\title{
A scalable and customizable multimedia framework for behavioral intervention
}

\author{
Diogo Fernando Trevisan ${ }^{1,2}$ (D) Fernanda A. B. de Araujo ${ }^{2}$ Priscila Benitez ${ }^{2}$. \\ João Paulo Gois ${ }^{2}$
}

Received: 9 December 2020 / Revised: 24 December 2020 / Accepted: 28 December 2020 / Published online: 13 January 2021

(C) Beijing Normal University 2021

\begin{abstract}
We present a novel web-based computational framework to aid behavioral interventions for people with autism spectrum disorder (ASD). The development of the framework aims on providing resources for creating, editing, storing, and applying multimedia activities, as well as tools for tracking and analyzing the students' progress. We have conducted the design and development of our framework based on principles from applied behavior analysis and behavioral psychology. We evaluated our framework, testing it with five professionals (participants) that freely explored it. During the tests, we tackled minor adjustments pointed by them, while the users' general suggestions are assisting us to further improvements. The participants answered two questionnaires about their profiles, as well as how they explored the framework. After the tests, we observed that the use of the framework can be positive for the interventions, considering both the activities created by each professional and their reports. Last but not least, the user tests were conducted, in Brazil, during the period of social isolation due to Sars-cov-2 pandemic, where the participants had the opportunity to explicit how technologies, in particular our framework, can benefit their work.
\end{abstract}

Diogo Fernando Trevisan

diogo@comp.uems.br

Fernanda A. B. de Araujo

fernanda.aparecida.araujo@hotmail.com

Priscila Benitez

priscila.benitez@ufabc.edu.br

João Paulo Gois

joao.gois@ufabc.edu.be

1 Computer Science and Information Systems States University of Mato Grosso do Sul - UEMS, Dourados- MS, Brazil

2 Present Address: Federal University of ABC - UFABC, Santo André - SP, Brazil 
Keywords Applied behavior analysis - Software design - Customizable teaching activity $\cdot$ Autism spectrum disorder (ASD) · Framework $\cdot$ Sars-Cov-2 pandemic . Social isolation

\section{Introduction}

The autism spectrum disorder (ASD) occurs in all racial, ethnic, and socioeconomic groups (CDC 2016), being four times more common among boys. The official health manuals (American Psychological Association 2019; World Health Organization 2020) identify characteristics related to neurodevelopment, with deficits in social communication, restricted interests, and stereotyped behaviors. The average prevalence of the number of people with ASD, identified in European, Asian, and North American studies is around 1\% to 2\% (CDC 2016). In the USA, it affects 1 in 54 American children, up to eight years old (CDC 2016; Maenner et al. 2020).

The treatments for ASD have been conducted in different formats, from medications to interventions - models of actions defined by professionals in the areas of health and education for the development of people with ASD, based on the definition of teaching objectives that aim to improve the quality of life. Medavarapu et al. (2019) investigated studies dealing with treatment modalities for people with ASD. The results showed evidence for the applied behavior analysis (ABA) and some drugs for specific cases. The Teacch Program and Denver were evaluated as "possibly bringing benefits" but without sufficient scientific evidence. Sensory integration was on the list of treatments that do not have a scientifically proven effect (Medavarapu et al. 2019).

The literature shows that intensive behavioral interventions are organized based on individualized stimulation. In this case, one professional for one person with ASD, with a workload of around 15 to 40 hours per week, for at least two consecutive years, starting from a varied curriculum built from the Behavior Analysis approach (Vivanti and Pellecchia 2020).

In the last decades, the use of behavioral intervention has presented the most acceptable results for children with ASD (Higbee et al. 2016). The use of ABA, through intensive behavioral intervention for people with ASD, was based on the pioneering study of Lovaas (1987). The purpose of the intervention is to improve the student's quality of life, through teaching activities that stimulate their development to prepare them for autonomy, in different contexts, such as school and, above all, social (Cooper et al. 2007).

In a behavioral intervention, an individual's undesired behavior is replaced by the desired behaviors through small activities. Each activity presents a situation, in which certain responses (behaviors) are expected. If the student's answer is correct, the consequence could be a preferred item, previously evaluated, e.g., a YouTube video or a candy. Otherwise, the consequence may vary according to the purpose of the established teaching procedure. A corrective consequence could be the re-presentation of the situation, along with a clue (or help) that favors the student to choose the correct answer. This way the student gains access to the preferred item (Cooper et al. 2007). It is necessary to verify which reinforcing 
stimulus will be provided to the student (based on the student's preference). The reinforcing stimulus refers to the one that increases the chance of the expected behavior happens in the future.

One can use different teaching procedures in behavioral intervention, such as incidental teaching, pivotal response training, and the discrete trial training (DTT) (Lerman et al. 2016).

A DTT, which is one of the most common teaching procedures, involves the following steps:

1. Attention: get the student's attention;

2. Antecedent: presentation of the initial instruction (may have materials, stimuli and some clue, when necessary);

3. Response: student's response;

4. Consequence: presentation of the consequence to the response, and;

5. Record: record the attempt, if the student answered correctly, wrong, or needed some tip.

Several teaching activities suitably fits the DTT format (Lerman et al. 2016). As a teaching activity, the behavior analyst can ask the student to draw, imitate gestures, drag objects, point, among other activities, as long as the activities are presented in the DTT format.

In a behavioral intervention, each student has a customized curriculum, composed of different teaching programs. Each teaching program aims to teach a specific behavior or knowledge through various activities that the student has to perform.

Traditionally, the activities are performed with physical materials, such as printed cards or toys. Then, the teacher needs to handle simultaneously these material and the attention of the student while recording the student's performance in paper forms. After the sessions, these collected data are, in general, compiled in some spreadsheet software by the teacher to infer the student's evolution, as well as for future decision-making. Thus, naturally, two challenges arise: the divided attention of the teacher during the sessions and the need to input the data in a digital spreadsheet.

The current work arises in the following context: the evidence of ABA to treat people with ASD (Medavarapu et al. 2019), the use of technological resources to foster the intervention process (Artoni et al. 2018; Chien et al. 2015; Dehkordi and Rias 2014; Dillon and Underwood 2012; Trevisan et al. 2019), the difficulty in finding easy-access computational tools, and the nonexistence of free flexible computational tools for customizing activities according to the student needs with the capability of providing resources to apply the activities and record the data (and produce reports) to posterior analysis.

Thus, in this study, we present a novel computational framework to aid in the workflow of the behavioral intervention for people with ASD. We evaluated our framework, asking the professionals to test and explore it, from their perspective, as a tool to help in the behavioral intervention, especially during the social isolation period of Sars-Cov-2 pandemic we have been facing in 2020. 
This paper is organized as follow: "Related work"presents the studies related to the use of technological frameworks for behavioral intervention to people with ASD; "The proposed framework" details the design of our framework; "The methodology" describes how we applied our user evaluation; "Results" relates our results; "Discussion" presents our discussion about our results, and; "Conclusions" concludes this study providing future works.

\section{Related work}

The use of technologies in education have been broadly investigated in the last decades, including those one for special education. Cheng and Lai (2019) presented a very broad Systematic Literature Review, selecting studies from 2008 to 2017, investigating how researchers have used technologies in special education, which includes autistic children. The found studies included the use of devices, learning strategies, learning domains, and research issues. The authors reported that the researchers used mobile devices, notebooks, personal computers, and tablets as tools in special education.

Trevisan et al. (2019) reviewed technologies used in behavioral intervention. The authors cataloged from scientific databases studies that have developed or applied technology to assist the behavioral intervention for students with ASD. Among the dominant technologies, the use of robots and applications developed specifically for an activity or for teaching a specific skill stands out. According to the authors, one of the main issues in the existing applications is the lack of customization of teaching programs. The existing software has predefined activities, and it is not fully customizable according to the needs of each student. Some software allows configuring activities; however, they are limited to selecting stimuli that will be displayed to students and with few activity models-usually only Matching-To-Sample (MTS) activities.

Chien et al. (2015) proposed and evaluated an app, named iCAN, that is a communication assistant and a tool to teach Picture Exchange Communication System (PECS). With the app, users can create new cards and share them with other users. The researchers applied 4-week tests to 11 children with moderate to severe autism, with low to no-verbal skills, ranging from 5 to 16 years old. The parents and caregivers reported that they had gained much time in creating the cards, in contrast to those printed with pictures. The app also allowed cards to be shared, so users produced content that everyone could use. The authors collected data from questionnaires with the study participants, who were primary school teachers.

Dillon and Underwood (2012) tested the use of computers so that autistic children could create stories. The study aimed to explore expressive writing skills as a measure of imagination. Using the Bubble Dialogue software (Cunningham et al. 1992), two story scenarios were presented: the first based on reality in which friends visit each other's home after school, and the second based on a fantasy scenario with aliens living on an undiscovered planet. The software featured a prologue to a story where the child had to select a character. Above the characters, there are balloons in which the child must insert texts to complete the stories. The studies were carried out in the children's 
homes being assisted by their parents. As expected, children completed stories presenting more errors in fantasy stories than those based on real life situations.

Dehkordi and Rias (2014) proposed an application for tablets to train the response to multiple cues. The application has a game-like interface, with different levels. For instance, a train is presented with different wagons where the student must assemble another one similar to the model. Another activity presents a road with different signposts, and the child must compare a vehicle with the signpost to decide which way the vehicle should go. The authors applied tests with six students aged from 5 to 8 years with ASD. After a few sessions, the students became interested in the application and to performed the simple activities correctly. The authors concluded that the use of rewards and games are motivating for students with autism.

Artoni et al. (2018) presented the ABCD SW, an ABA based system. The software, accessed from the web, was developed using participatory design. The software allows the behavior analyst to create Matching-To-Sample activities and apply them with students. It is also possible to view reports and monitor the individual development of each student. The authors applied tests with 7 children with autism and a workload of 25 to $29 \mathrm{~h}$ per week. Using the software during therapy made the sessions faster and also allowed creating activities and collections easier. The software also made the children pay more attention to the activities. The ABCD SW allows creating MTS activities with personalized stimuli. However, the positioning of the stimuli is fixed and the activities that can be created are limited to MTS.

Dunn et al. (2017) present MITA, an app with many activities, including puzzles, matching, patches, and outlines. The authors tested the software over the internet, distributing the app for Android and iOS, while tracking the results remotely. Based on the observed data (hits and misses), the authors stated that the children could learn from the proposed activities. This software does not allow the user to create activities, and the application selects which activity will be presented to the student.

All around the world, to face the Sars-Cov-2 pandemic isolation, educators sought online tools to create activities for the student. Not every tool was designed to create activities or to record the activity data. Very common tools such as presentation programs (Stenhoff et al. 2020), which were not designed to create activities and do not record automatically the student answers, are being used by the educators to create online activities. The students can then perform the activities at home, with the help of their parents or tutors.

We designed our framework to help educators create, apply, and manage teaching activities and to track student's progress with an easy-to-use activity editor, configurable templates, and tools for reporting. "The proposed framework" presents the design process of our framework and how it can be used to configure different activities.

\section{The proposed framework}

\section{The design process}

The motivation for the development of our framework arose from the need of the ABA community for a software that would assist in their interventions (therapies). 
We have raised the core requirements for our framework through meetings between the design team, which includes professionals from computer science, education, and psychology. In these meetings, it was possible to verify what features a software (or framework) should have to ease the work of educators and psychologists, providing more autonomy and efficiency. We classify these meetings into three categories:

- Meetings with developers and behavior analysis researchers who are currently using computer systems;

- Meetings for observing the application of ABA therapy;

- Internal meetings among the developers and psychologists for design and developing the present framework.

We had meetings with developers and behavior analysis researchers who are currently using computer systems in their interventions and researches, specifically, the software GEIC (de Souza Junior et al. 2012) and Equiv (Pimentel et al. 2009). Both software have been used widely by behavioral analysis professionals in Brazil; however, they were designed previous to the current tendencies in Human-Computer Interaction, as well as the evolution of smartphones and social networks. Particularly with GEIC, professionals can configure MTS activities and anagrams. However, according to the professionals' comments, the software is difficult to use, demanding many steps to configure an activity-they would like to configure activities with few steps. When configuring a teaching program, the settings are not intuitive as the user interface presents many elements simultaneously. Equiv's user interface is cleaner than GEIC but the activities that the user can configure are also limited to MTS and anagrams. Besides, both GEIC and Equiv do not allow inserting videos, do not generate very detailed reports, and run only on desktop computers. One need pointed out by professionals is that the software should run on mobile devices in which the students and parents could access it from home.

To understand the workflow of behavior analysts, the developers participated in ABA sessions. During the observations, it was possible to verify the dynamic of an ABA session. In one session, the professional usually brings a bag of toys to be used as activities and rewards, as well as sheets where the teaching schedule is described and where the professional will record the session data. After the session, the professional then digitizes the data into a spreadsheet processor to keep the history and to analyze the evolution of the students. We observed that during the sessions students have shown interest in technology not only as a reward but also as a tool to perform activities. For instance, during a session, a student preferred to control a mini-robot with a screen as a face than his favorite reward (a snack). The student is hyperactive but controlled the robot through a smartphone easily and fulfilled the activities that the educators asked him (Trevisan et al. 2018).

After the meetings with users and developers, and the observation of interventions, we started the development of our framework. During the design of our framework, we focused on ensuring three key features: universality, the capability to be easily incorporated into the workflow of psychologists and educators, and the capability of generating reusable resources. Specifically, with our framework, the users can create their activities from new templates as well as create 
new templates for future activities. We consider our application as a framework because it is customizable, where users can create their activities as well manage them, although experience with computer programming is not required.

All the meetings indicated that our framework should run on a web server with support for multimedia data. First, the users do not need to install the software on their computers, smartphones, or tablet. Second, web-based software can run on any device compatible with HTML5, this includes computers, smartphones, and tablets. Third, it makes the software scalable, where the psychologists and educators can easier handle the therapy of many patients.

When the software development advanced to a level that we could test it, we asked the participants (user testers) to test and evaluate the framework. The evaluation is presented in "The methodology". During the tests, we received the participants' feedback. With that feedback, we added the most immediate updates to our framework. We summarize our development cycle in Fig. 1.

In the current version of our framework, the users can create MTS activities, text filling, dragging, and activities that students perform outside (such as imitation of gestures or pointing). It allows the user to record all attempts, facilitating the professional's workflow. Regarding the users' profile, the framework provides two: one for the professional and another for the tutor. A professional manages curriculum and activities. Besides, the professional can register tutors, who can apply the teaching programs. Figure 2 presents the different roles for each user (professional or tutor).

Using our framework, professionals can create new activities, create customized rewards, configure complete teaching programs, and view reports. The following sections describe the features implemented in the framework.

\section{Activities}

In our framework, each activity comprises different screens that will be displayed to the student. One screen could be, for instance, the display of an image or video. During the development of our framework, the team defined a set of basic screens. One activity can have one or more screens and each screen have its behavior. The screens defined in our framework are:

- Display stimuli: stimuli are displayed for a user-defined period. The supported stimuli types are images, texts, audio, and YouTube videos;

- Select stimulus a model stimulus (optional, which can be an image, audio, or text) is displayed. The student has to compare the model with other displayed stimuli and select one of them. This screen is used to create MTS activities;

- Fill text stimuli are displayed along with a text box in which the student can write;

- Drag the student can drag images and place them on top of others (drop), and;

- Tutor's Feedback allows tutors to provide feedback for one activity performed outside the framework; 


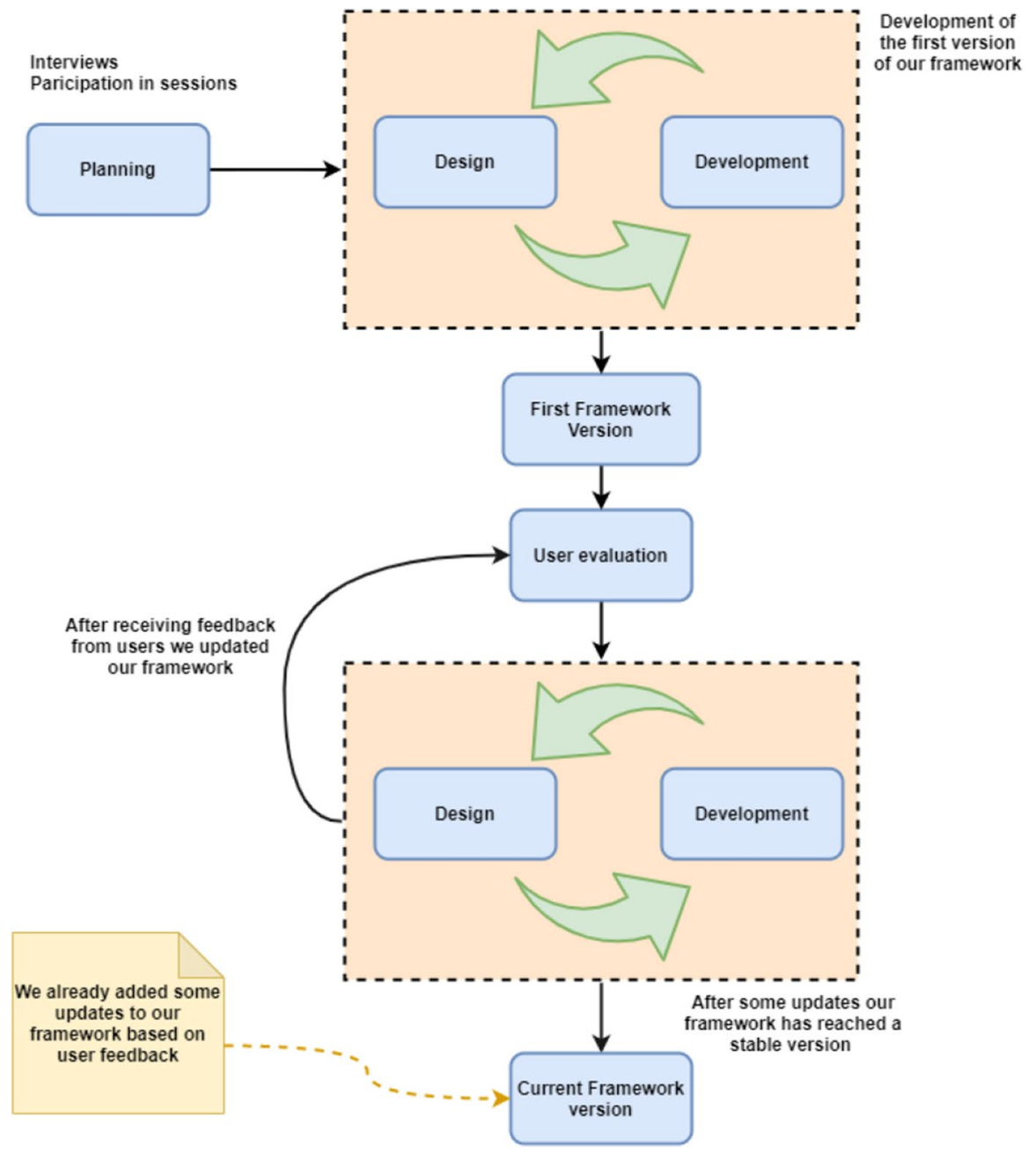

Fig. 1 Our design process: we started the design by interviewing professionals and watching ABA sessions. After collecting the user requirements, we started the design and development of our framework. Later, during tests, after users' feedback, we added updates to our framework. Currently, we have a stable version available for use

Our framework provides, by default, templates of activities that use the screens. Those default templates are accessible by all users, and the users can use them to configure their activities. For future versions of our application, we can add new default templates whenever the users ask, and whenever we agree that this template could be useful for other users. Also, the professionals can configure their activity templates using the activity screens, and they can share those templates. The default templates in our framework, at the time of writing this paper, are: 


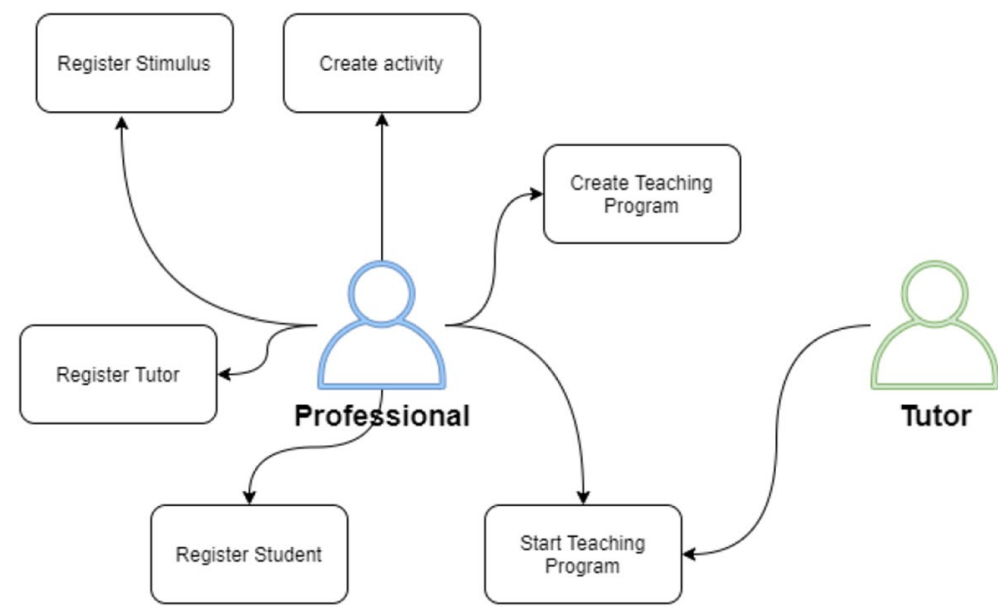

Fig. 2 User role in our framework: professionals can register stimulus, register students, register tutors, create activities and teaching programs, and start teaching programs (intervention); tutors can only start the teaching program with students

- MTS: MTS activity, where one model stimulus is presented and the student should select another stimulus that matches the model. It is built using one "Select Stimulus" screen;

- Dragging: an activity where students should drag images. This template is built using one "Drag" screen;

- Instruction: activity to ask the student to perform some action in the real world and record their answers. In this template, we use one "Display stimuli" screen followed by one "Tutor's feedback" screen;

- Text Input: students can insert (type) text. This template only uses one "Fill text" screen;

- Delayed MTS (D-MTS): this activity presents the model stimulus and waits with a blank screen; then the comparison stimuli are presented to the student, who should select one stimulus. The D-MTS template is build of one "Display stimuli" screen followed by one "Select stimulus" screen, and;

- Sequencing: given a numerical or alphabetical sequence, the student should fill in the missing characters. This template is built using one "Drag" screen;

The possibility to create a custom activity or templates increases the customization of students' curricula. The activities created can be stored as a single activity, to be used once, or an activity template, to be used to create several activities.

In ABA, a concern is the reward that will be presented to the student. Researchers performed tests to define what are the children's preferred rewards. Among the raised options, children like to see pictures, fireworks animations, and familiar sounds, such as the voice of their parents (Constantin et al. 2017). Our framework allows the professional to create customized rewards for each student. As a reward, the educator can present images, texts, sounds, or videos. 


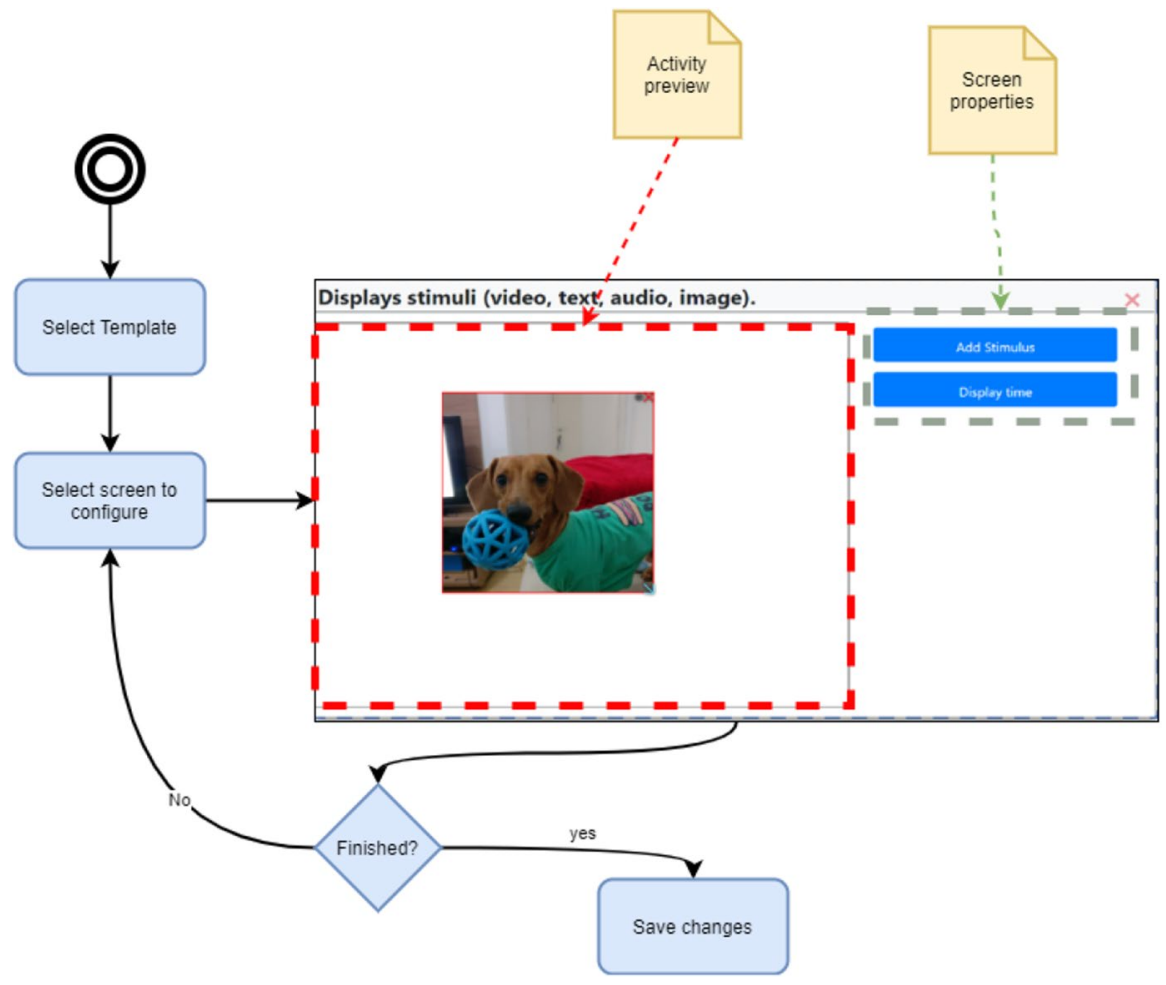

Fig. 3 Activity editing screen: users can select one screen that will be displayed to the student and edit its properties. In this example, it is possible to add stimuli to be displayed and configure the time the screen will be shown to the student

Figure 3 shows the activity configuration. Here, the user can see the activity screens that compose the activity. By clicking on one screen, the editor is open, and the user can see the settings available. Also, users can preview how the stimuli will be presented to the students.

\section{Configuration of teaching programs}

Professionals organize the activities they created as Teaching Programs. Our framework has an interface to add activities and configure their consequences in case of the correct answer (display reward) or in case of wrong answer (corrections). If the student answers an activity correctly, it is possible to display a customized reward, which may include images, videos, texts, or audio. If the student provides a wrong answer for an activity, it is possible to present a configurable tip. The professional can also configure which activity will be performed in case of success or error, providing a correction if the student answers an activity wrongly.

Figure 4 shows the interface for configuring a teaching program. The professionals can add different activities to create the trials and configure its consequences and the 


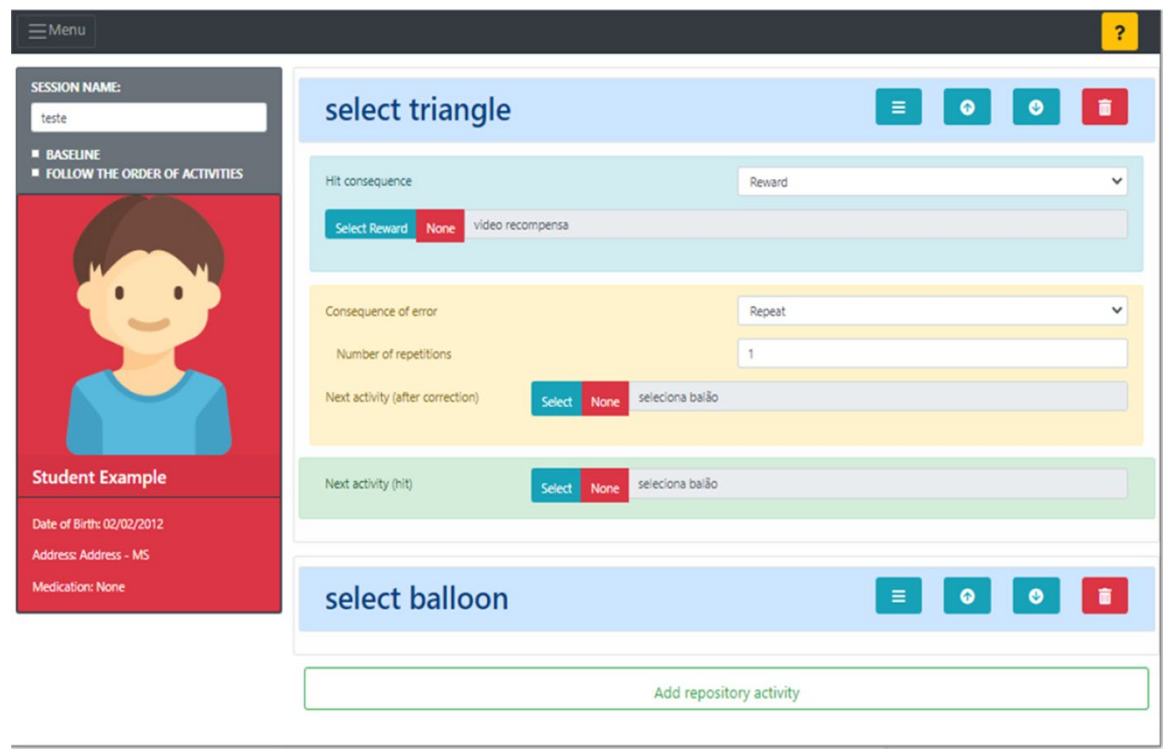

Fig. 4 Configuration of the teaching program: for each activity, it is possible to configure which is the next activity in case of success or error and the consequences for the activity

next activity. It is also possible to mark a program as a "baseline", which performs all activities in order without displaying corrections or rewards, just for evaluation. It is also possible to mark an option to follow the order of the activities so the professionals do not need to configure the next activity in case of correct or wrong answers.

\section{Reports}

Every trial is recorded in a database, allowing the professionals to access these data from reports. For the activities that students perform outside the framework, the evaluation is provided by one tutor (parent, teacher, or professional). The professionals can check student's progress by Teaching Program or by Activity. Figure 5 overviews the report for a Teaching Program, with the percentage of correct answers, and the history of correct answers and errors over time. It is also possible to check the results of each trial of the teaching program, showing the name, the stimuli used, which stimulus the student selected, and the result of the trial. Professionals can check reports per activity, showing the student's evolution. Also, the drag activities show an image with the final configuration of stimuli, after the student has dragged them. 


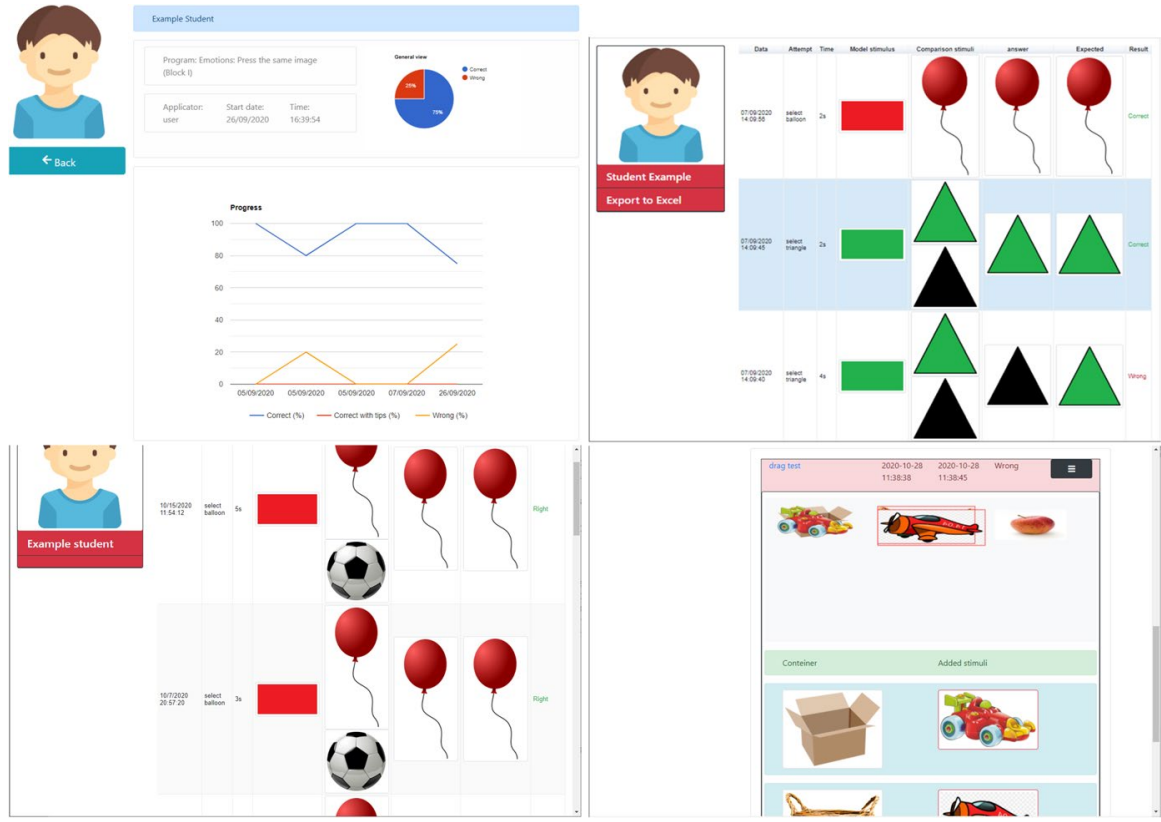

Fig. 5 Reports in our framework: users can see the student's evolution over the same teaching program (top left), check the stimuli used in every trial (top right), check the history for a specific trial (bottom left), and the screen for the drag activity (bottom right)

\section{The methodology}

\section{Research design}

Because of the social isolation of Sars-Cov-2 pandemic, we could not conduct classroom-style meetings to train the participants. Then, we provided online video tutorials to help them to learn how to use the framework. We also answered their questions by e-mail or by a WhatsApp group. Some questions or feedback, which were minor issues, defined the first set of tasks we employed to improve our framework.

We elaborated two online questionnaires to evaluate our framework. The selected participants are professionals working with behavior therapy. We invited them to participate freely and voluntarily after reading and signing the consent terms. The aim of each questionnaire was:

\section{General profile of the participants}

The first questionnaire aimed to identify the profile of the participants. We applied it before the use of the framework, containing the following information: age, gender time of professional activity, and computational knowledge. We also investigated if the participants had already used software in their interventions before or during social isolation because of the Sars-Cov-2 pandemic. Table 1 provides the participants' profile, whereas Table 2 presents whether the participants have used software 
Table 1 Characterization of participating professionals

\begin{tabular}{llllll}
\hline & Age & Gender & $\begin{array}{l}\text { Computer } \\
\text { knowledge }\end{array}$ & $\begin{array}{l}\text { Time working with } \\
\text { behavior intervention }\end{array}$ & $\begin{array}{l}\text { Previously used software } \\
\text { to perform interventions }\end{array}$ \\
\hline P1 & $31-40$ & F & Yes & 6 years & No \\
P2 & $31-40$ & F & Yes & 1 year & No \\
P3 & $20-30$ & F & Yes & 2 years & Yes (smartphone games) \\
P4 & $20-30$ & M & Yes & 1 year & Yes (computer) \\
P5 & $20-30$ & M & Yes & 5 months & Yes (MS PowerPoint) \\
\hline
\end{tabular}

in their interventions or not. Also, we asked questions to identify which features a behavioral computer application for interventions should have and what activities the application should have. The participants' answers are presented in Table 3.

\section{Framework evaluation questionnaire}

Applied after the use of our framework and the programming of activities for behavioral intervention. The questionnaire contained nine specific questions about our framework and has the objective to evaluate the participants' experience with the framework, assessing its functionalities, difficulties, limitations, and benefits. We discuss the participants' answers in "Results".

\section{Participant profiles}

We invited professionals working with behavioral interventions to assess our framework. The goal was to develop Teaching Programs for remote applications. In short, we aim to evaluate whether our framework is viable and receive feedback for further improvements, which were not detected by the development team. Table 1 describes the profile of each participant.

\section{Results}

Our questionnaires foresaw to understand the professional working with $\mathrm{ABA}$, their experience using technology, aspects from the evaluation of our framework, and specific questions related to the current period of social isolation. Figure 6 shows the questions grouped by thematic axis.

We grouped the results analyzing the general axis and the specific axis. The analysis of the general axis is presented in Table 2 regarding the variable of social isolation and professional work using applications.

The analysis of the general axis data (Table 2) refers to the use of computer applications in the period prior to the social isolation, on a general perception of participants considering other applications used in their professional practices and possibilities for improvement. Regarding the limitations of previous applications used by the participants, P4 reported "JECRIPE was not exactly developed 


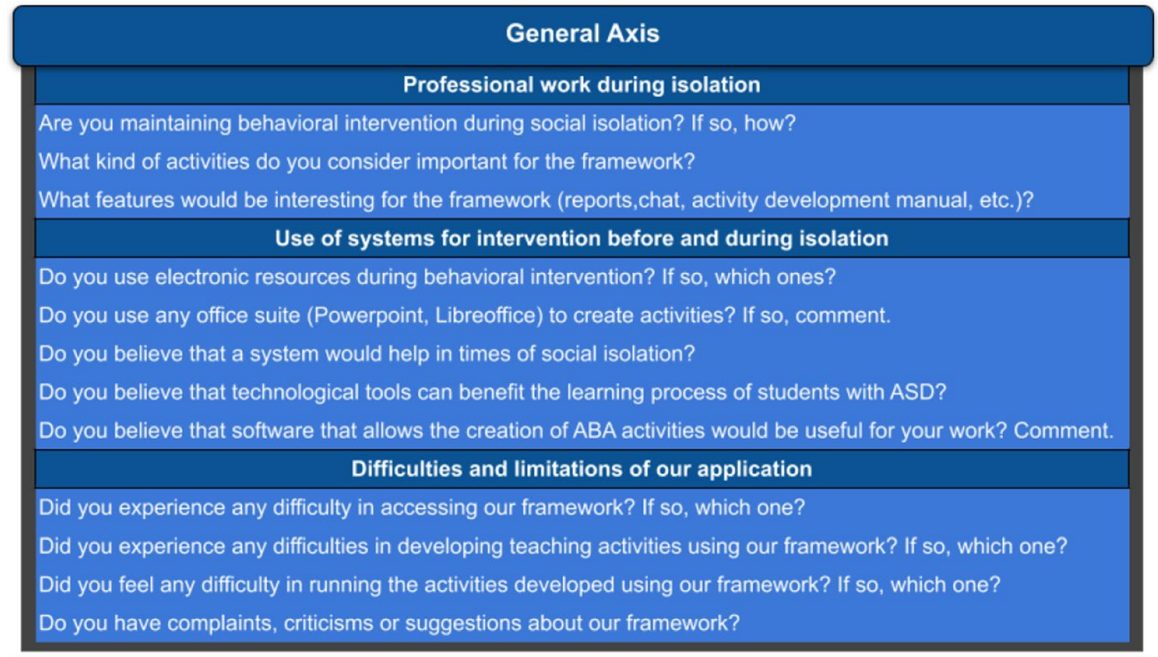

\begin{tabular}{|c|}
\hline Specific Axis \\
\hline Numerical assessment of our application (1-10) \\
\hline $\begin{array}{l}\text { From } 1 \text { to } 10 \text {, what grade would you give to our framework? (Considering } 1 \text { as bad and } 10 \text { as great). } \\
\text { From } 1 \text { to } 10 \text {, how do you evaluate your experience with our framework? (Considering } 1 \text { as bad and } 10 \text { as } \\
\text { great). }\end{array}$ \\
\hline Benefits of our application \\
\hline $\begin{array}{l}\text { Would you use our framework again in your interventions? Do you believe that the interventions carried out with } \\
\text { the framework would generate results with students? }\end{array}$ \\
\hline $\begin{array}{l}\text { Briefly describe the process of using the framework in the interventions with students (exploratory phase, } \\
\text { adaptation and use of the framework). }\end{array}$ \\
\hline
\end{tabular}

Fig. 6 Questionnaire: we divided the questions in two axis, one being general about participants' characterization and another specific one to evaluate the participants' experience with our framework

according to the molds of the universal design, leaving some important points such as better lighting, larger letter sizes, among others, without the possibility of adjustment. We use mTEA very few, but one limitation was an advance of

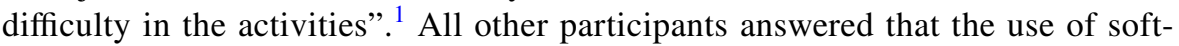
ware to create activities would be important to optimize professional performance throughout the behavioral intervention.

Regarding the benefits of how a computer application would help in interventions, P1 said "It provides speed for scheduling activities, making it possible to prepare many tasks in a timely manner. And for the student it is attractive to carry out activities using the computer, especially for those who don't have or have little access at home". P5 answered "It allows more possibilities and varieties of activities for patients". Other participants said that a computer application for interventions would be very useful. Table 3 shows what activities and functionalities each participant expects from a computer application for behavioral

\footnotetext{
${ }^{1}$ All speeches were freely translated from Brazilian Portuguese. JECRIPE is an educational game (Brandão et al. 2010) and mTea is a tool for configuring MTS activities that the participant had used.
} 
Table 2 Isolation, professional work and applications

\begin{tabular}{llll}
\hline & $\begin{array}{l}\text { Professional activi- } \\
\text { ties during social } \\
\text { isolation }\end{array}$ & Use of software in behavioral interventions & \\
\cline { 3 - 4 } & Previous to isolation & $\begin{array}{l}\text { During } \\
\text { isola- } \\
\text { tion }\end{array}$ \\
\hline P1 & Yes & No & No \\
P2 & No & No & No \\
P3 & Yes, through online & Yes, used smartphone games & No \\
& guidance with & & \\
parents & No & Yes, used the game JECRIPE and the software MTEA & No \\
P5 & Yes & Yes, used PowerPoint to create stimuli through images & No \\
\hline
\end{tabular}

interventions and which features a new computer application should present. We applied this questionnaire before the participants have tested our framework.

The participants evaluated our framework, relating their difficulties and potential limitations. P1 tried to use the framework without watching the video tutorials. P1 presented difficulties on the first access: "On the first access, it was crashing on the third screen". We don't know what happened, but maybe P1 experienced a server problem. Also, P1 faced issues finding some links and buttons on the computer screen, but, after watching the tutorials, it became easier to find the buttons and links. P1 suggested that the framework should have an option to configure consequences when the students answer wrongly to the activity but the option is already in the framework. The screen that refers to is shown in Fig. 7. On this screen, the user can configure a reward for correct answers and corrections in case of a wrong answer.

The participant $\mathrm{P} 2$ was not capable of creating activities in her/his first time using the framework, but, after watching the tutorials, it became easier. P2 suggested ("I) load activities faster when it is the baseline. 2) Be able to select the activities you create for a student for another so you do not have to register each activity. 3) have a button to return to the student area". We already optimized the loading time and users can now copy the teaching programs from a student to another ((reuse)). Also, we added buttons to return to the students' main screen.

Participant P3 experienced difficulties in saving stimuli and grouping images. As suggestions, $\mathrm{P} 3$ answered "Possibility to enter learning criteria. Repeat the training until the student can develop independently". Participant P4 reported that the transition time between activities was too high and "the system needs steps to return to some previous point. Especially in the edition of the teaching program to see how it will be presented for the student-the edition is done in one screen. Sometimes finding the desired loaded stimulus is a little difficult, since, in my case, most start with the same name, and, sometimes, I forgot how I saved it". The teaching program edition is presented in Fig. 7. To start a teaching program, the professional needed 
Table 3 Expectations on which activities and functionalities a computer application for ABA should have

Desired activities

P1

P2

P3

P4

P5

Basic abilities and academic skills (literacy and mathematics)

Identification of numbers, quantity, matching of shadow, matching of figures

Academic, identification of figures among others

Drag the object to the right location, choose the right option, sequence a story, imitation

The more variety the better, example, identification, matching and naming, and others with markings on a stimulus, game of seven errors (difference game), find the objects or find the absurd

Suggestions for a new application

P1

Reports that allow daily monitoring of student development, with automatic graphics generated by the system

P2 Complete report, baseline for identification of what the students knows or not, activities designed according to the student's needs

P3 Reports, chat, activity development manual

P4 System tutorial, intuitive interface, reports, being able to drag materials from the computer itself to the system, the activity developers could share their work to leverage collaborative teaching (perhaps a forum), activity bank and presentation of a report with the mistakes and the response time of the teaching programs

Manual, reports, artificial intelligence that modifies activities based on learning criteria, editing tools to create activities similar to those of power point, option to copy and paste programs to facilitate work, options to order and organize activities, reinforcements and stimuli created

\section{basketball game}

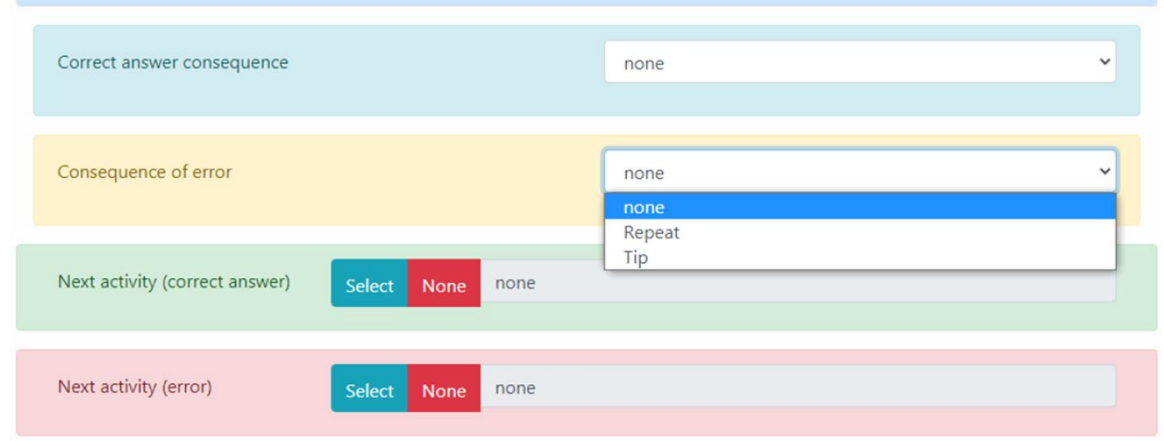

Fig. 7 Configuration of consequences: users can select the consequence whenever the student selects the correct or wrong answer for an activity 

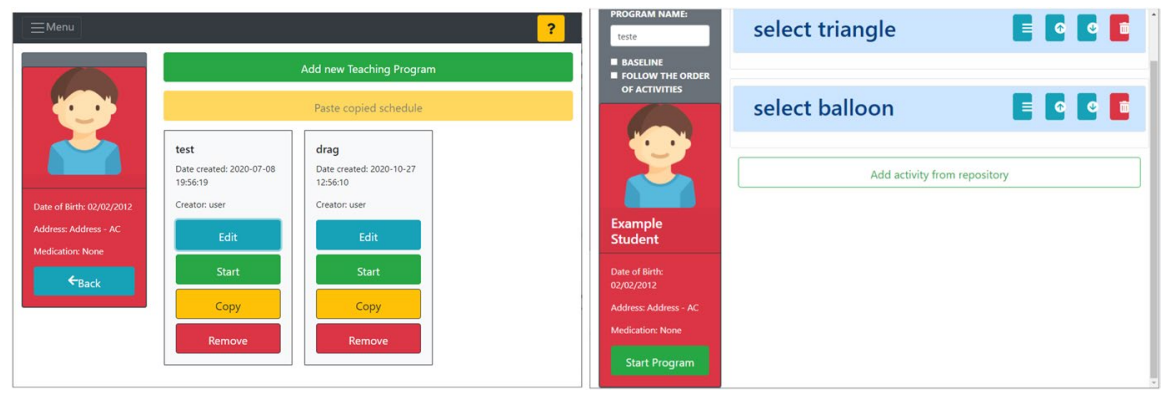

Fig. 8 Teaching programs: to start a teaching program the user needed to return to the students' profile (left) but now professionals can start the program on the edition screen (right)

return to another screen as shown in Fig. 8, but currently, we added a button to start the teaching program on the Teaching Program edition screen.

We already tackled the high loading time faced by P4. As suggestions, P4 answered "The system could not always oblige you to upload the stimulus, allowing you to drag it from the computer, and put it in the activity; if this is not possible, let us create folders for use within the system, and let it appear at the top of the stimuli suggested for the user". To use a stimulus, the user first needs to register it. P4 said that it would be better to use without registering, but the stimuli registration is necessary because they can be used again in other activities and to categorize the activities in future work.

Participant P5 was the one who created the largest number of activities, using our framework deeper. As difficulties, P5 said "Some templates need more buttons to insert audio instructions like the dragging template. Allow the insertion of more than one model stimulus, as sometimes it requires an image and instructional audio. Place the stimuli of equal size when creating an activity or place them aligned and well distributed on the screen'. P5 provided us richer suggestions: "Sometimes the reinforcement takes time to appear; I believe it is related to the internet connection and perhaps other factors; ". We already tackled these comments, loading the entire session at once. P5 also argued "More stimulus editing tools could be inserted in the program itself (cut, copy and paste, to an exact size, resize only one side of the stimulus, stimulus alignment tools). If possible, being able to copy ready-made programs from one student to another to speed up the registration of activities". We are aware about these features, where the feature to copy programs was already inserted in the framework, and tools to edit images will be considered in the next version of the framework.

Both participants P1 and P2 argued that they never needed to use any software in their interventions before or even during social isolation because of Sars-Cov-2 pandemic. Along with the test of our framework, P1 experienced a few problems to access the framework and later to find information on the screens. However, after watching the tutorials, P1 could use the framework without issues. P2 had some issues adding stimuli to the framework, but, again, after having watched the tutorial videos she/he could add the stimuli. 


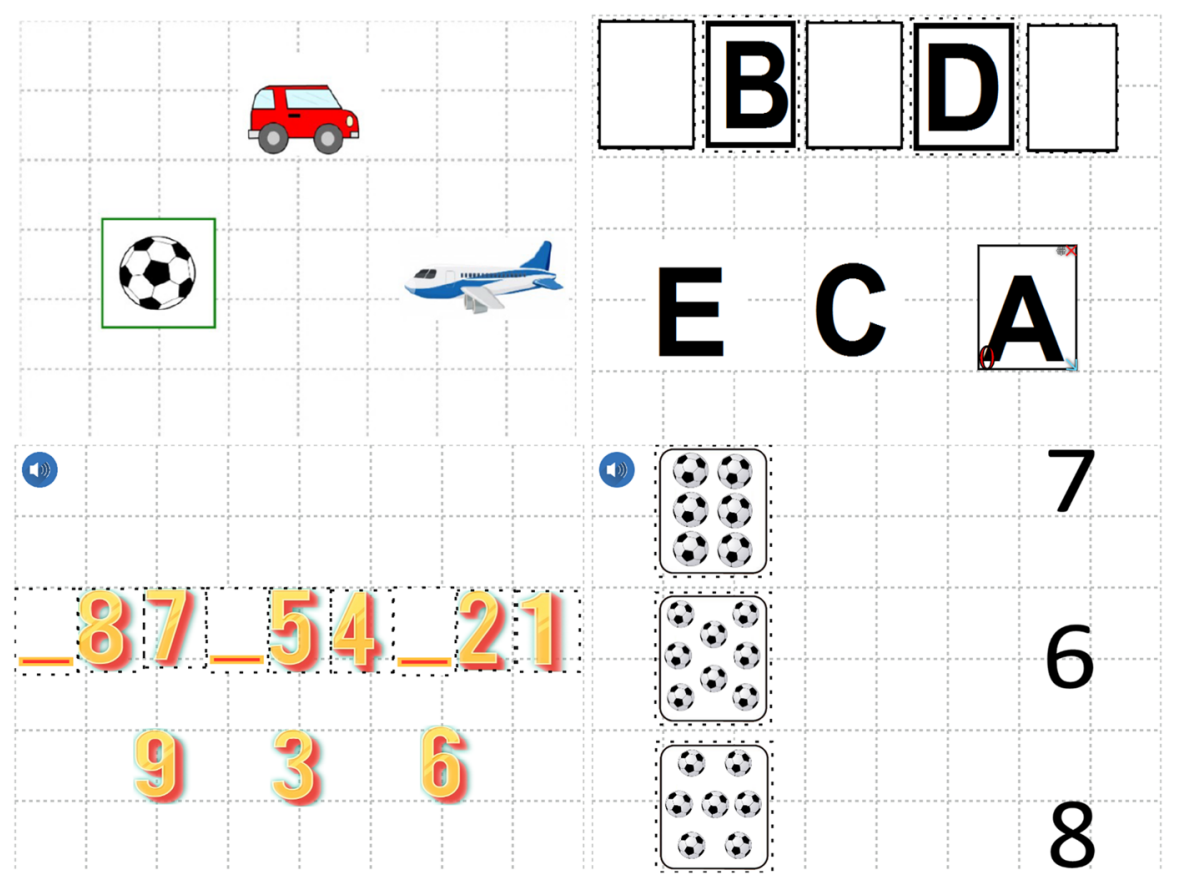

Fig. 9 Illustrations of Activities created by participants: Auditory-visual pairing (top left), dragging-tosample (top right), numerical sequencing (bottom left), and dragging-to-sample with numbers (bottom right). The "audio" icon indicates that the activity has a sound stimulus

The Participants P2, P4, and P5 reported about the transition time between activities. P4 said "Sometimes the system takes a while to show the stimuli, which at first made me believe that it had not uploaded it correctly". The framework was loading the activities one-by-one. Currently, the framework does not have this feature. The entire session is fully loaded, then the transition between activities is very fast.

Figure 9 illustrates examples of experimental activities programmed by participants. For instance, P1 proposed an audio-visual pairing activity, using three images, in which the student is instructed orally to select the ball. This type of activity is important to teach the relationship between the name of the object and its referent, thus expanding the receptive vocabulary of the student. P2 developed a dragging-tosample activity, which consists of dragging the correct letter to complete the alphabetical sequence. In this activity, is expected from the student to learn the sequence of letters, according to the alphabet. P4 performed a numerical sequence activity by identity, which consists of the presentation of a complete sequence, where the student is instructed to compare by physical similarity the numbers presented as a model to complete the sequence in ascending order involving numbers from 1 to 9 .

Participants P1, P2, P4, and P5 created several activities. Only P3 has created no functional activity, only some tests. P1 created MTS and Delayed-MTS activities to identify parents and to train memory. P2 created MTS activities to teach letters and 


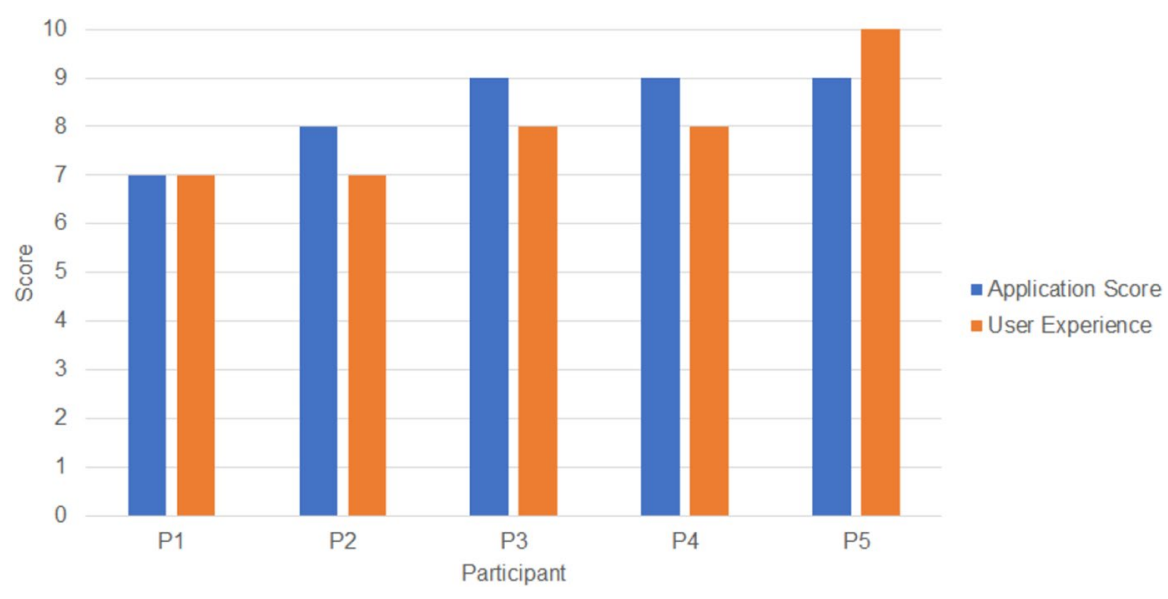

Fig. 10 Framework score: users evaluated our framework in relation to their performance compared to their expectations and personal experience using the framework. Framework's performance is presented by the blue bars and user's experience by the orange bars

numbers. P4's activities were based on MTS to teach number sequencing. P5 created activities based on dragging.

All participants answered they would return to use our framework during their professional practice and stated that the framework would be beneficial in the face of the social isolation resulting from the Sars-Cov-2 pandemic. This indicates the effectiveness of using the framework in programming activities to carry out behavioral interventions. Figure 10 presents the scores provided by the participants to the framework's performance based on what they expected from our framework (Application Score) as well as how were their experiences using the framework (User Experience).

\section{Discussion}

In this study, we presented and evaluated our framework from the perspective of professionals who apply ABA to people with ASD. Participants suggested improvements, for instance, in the design of the graphical user interface (GUI), and in the speed of activity transition. We found that the framework has great potential to be incorporated in the workflow of the professionals, assisting in the teaching of students, as indicated by the results of evaluations. We considered to the framework the same workflow and terminology the professionals who assisted us in the design are used to.

One feature of our framework relies upon the range of activities the users can create. In general, available software provides only defined teaching programs for specific behaviors, without the possibility of customization. Our framework advances this discussion by proposing diversified activities that can be customized by the professional as shown in Fig. 9, as well as the possibility to create custom templates. 
Table 4 Participants' expectations on activities and functionalities currently presented in our framework

\begin{tabular}{lc}
\hline Activities & $\checkmark$ \\
Basic abilities and academic skills & $\checkmark$ \\
Identification (numbers, quantity) & $\checkmark$ \\
Matching (shadow, figures) & $\checkmark$ \\
Drag the object to the right location & $\checkmark$ \\
Sequence a story & $\checkmark$ \\
Imitation & X \\
Naming & $\checkmark$ \\
Game of seven errors (difference game) & $\checkmark$ \\
Find the objects or find the absurd & $\checkmark$ \\
Functionalities & X \\
Reports & $\checkmark$ \\
Baseline & $\checkmark$ \\
Chat & $\checkmark$ \\
Tutorial & X \\
Activity bank & \\
Artificial intelligence that modifies activities based on learning & \\
criteria & \\
Editing tools to create activities & $\checkmark$ \\
Option to copy and paste programs & $\checkmark$ \\
Options to order and organize activities, reinforcements and & $\checkmark$ \\
\hline & \\
\hline
\end{tabular}

Regarding the behavioral intervention, it would be impracticable to use an application to teach each target behavior, without the possibility of customizing activities. Thus, an application that can involve multiple possibilities of activity customization is fundamental in behavioral intervention, which requires a variety and diversified teaching curriculum to serve all students with ASD. Our framework allows us to create fully configurable activities: the user can add stimuli, position, and resize them freely on the screens we defined. Our framework also allows one to configure rewards, corrections (repetitions, tips), and create activities of different types, such as MTS, real-world activities (such as imitation), filling in texts (to create stories), and activities where the student needs to drag stimuli.

Considering the questionnaires applied before the tests, we can list some user requirements that our framework already provides. Table 4 shows the participants' expectations on activities and functionalities they aimed from a new ABA computer application.

The participants were able to use our framework and created several activities. Improvements pointed out by them, such as optimizing the loading and shortening the interval between activities and the option to copy teaching programs have already been implemented and added to our framework. Other suggestions such as the game of seven errors (difference game) activity will be inserted in the framework in future updates. 


\section{Conclusions}

We presented the design, implementation and evaluation of a framework to assist the behavior intervention. The motivation for the development of this application was the treatment of ASD; however, it can be used by professionals from different areas, e.g., in staff training (Sarokoff and Sturmey 2004) or in classes with students with no special needs (Robacker et al. 2016).

The existing applications, presented in related work, do not allow a broad customization. Besides, if a behavior analyst aims to use a computational device in ABA therapy, one will probably need to use more than one application. In this case, one will need to collect data from each application and process them manually.

Our framework allows users to create activities with different predefined templates and the customization of new templates, providing a wide range of activities. Furthermore, our framework also allows using different stimuli, e.g., images, audio, text, and videos.

A contribution of our framework is its low cost and scalability. As it is webbased, one only needs to host it on a web server, allowing the access of many users. The activities generated by the framework can be accessed using smartphones, tablets, or computers. Professionals can create customized teaching curricula, where parents or guardians can apply them at home. The professionals can remotely monitor the progress of students.

The need for a web-based and customizable teaching system has become more evident during the Sars-Cov-2 pandemic. Educators had to create online resources for students, and a system that allows customizing activities and permits the students to perform their activities at home has shown to be essential.

The Behavior Analysis advocates the customization of students' tasks based on their performance in specific behavioral tasks, which improves their behavioral repertoire. Following this principle, our work addresses conditions for the programming of teaching, based on elements of the universal design of learning, which means innovating how we propose a programmed and personalized instruction for all people.

In future work, we are going to create more activity templates, such as image and video feedback and eye-tracking-based activities (Bradshaw et al. 2019). We have been working on a template for facial recognition to create activities for facial expression imitation, as this is an important task that children with ASD should learn (Trevisan et al. 2020). Another type of activity is to allow the student to draw on the screen. This could be used as a reward or as an activity, like tracing or mazes. We will also create other types of screens, so the users could create their own templates.

We are planning to evaluate our framework with students and their relatives to test the framework acceptance and receive further feedback for improvements. The collected data will be used to create activities automatically by deep learning techniques, as well as to check how the students are learning with the use of learning analytics (Siemens and Baker 2012; Manske et al. 2019). We aim not 
only to improve the reports but also provide interactive visualization tools, using techniques from visual analytics (Yuan et al. 2020) to generate significant graphics that help behavior analysts on decision-making.

Acknowledgements We would like to thank everyone who participated in this study, especially the participants from the "GPEEI - Grupo de Pesquisa em Educação Especial Inclusiva da UFABC" research group.

Funding This paper was financed, in part, by CAPES - Coordenação de Aperfeiçoamento de Pessoal de Nível Superior [Finance Code 001] and FAPESP - Fundação de Amparo à Pesquisa do Estado de São Paulo [Process Number 2019/25795-2].

\section{References}

American Psychological Association. (2019). Publication Manual of the American Psychological Association. American Psychological Association: Publication Manual of the American Psychological Association.

Artoni, S., Bastiani, L., Buzzi, M., Buzzi, M., Curzio, O., Pelagatti, S., et al. (2018). Technologyenhanced aba intervention in children with autism: A pilot study. Universal Access in the Information Society, 17(1), 191-210.

Bradshaw, J., Shic, F., Holden, A. N., Horowitz, E. J., Barrett, A. C., German, T. C., et al. (2019). The use of eye tracking as a biomarker of treatment outcome in a pilot randomized clinical trial for young children with autism. Autism Research, 12(5), 779-793.

Brandão, A., Brandão, L., Taveira, G., Moreira, B., Vasconcelos, C., and Clua, E. (2010). Jecripe: stimulating cognitive abilities of children with down syndrome in pre-scholar age using a game approach. pp. $15-18$.

CDC. (2016). Data \& statistics on autism spectrum disorder. Retrieved 29 June, 2020, from https://www. cdc.gov/ncbddd/autism/data.html.

Cheng, S.-C., \& Lai, C.-L. (2019). Facilitating learning for students with special needs: A review of technology-supported special education studies. Journal of Computers in Education, 7(2), 131-153.

Chien, M.-E., Jheng, C.-M., Lin, N.-M., Tang, H.-H., Taele, P., Tseng, W.-S., et al. (2015). ican: A tablet-based pedagogical system for improving communication skills of children with autism. International Journal of Human-Computer Studies, 73, 79-90.

Constantin, A., Johnson, H., Smith, E., Lengyel, D., \& Brosnan, M. (2017). Designing computer-based rewards with and for children with autism spectrum disorder and/or intellectual disability. Computers in Human Behavior, 75, 404-414.

Cooper, J., Heron, T., \& Heward, W. (2007). Applied behavior analysis. Upper Saddle River: Pearson/ Merrill-Prentice Hall.

Cunningham, D., McMahon, H., \& O’Neill, B. (1992). Bubble dialogue: A new tool for instruction and assessment. Educational Technology Research and Development, 40(2), 59-67.

de Souza Junior, G. N., Monteiro, D. C., Pereira, A. B. C., Barros, E. S., and Marques, L. B. (2012). Learning machine as an aid tool in behavioral analysis in the teaching of reading. (Máquina de aprendizagem como ferramenta de auxílio na análise comportamental no ensino da leitura). RENOTE, 10(1). Original document in Portuguese.

Dehkordi, S. R. \& Rias, R. M. (2014). Using mobile game application to teach children with autism spectrum disorder (asd) multiple cues responding: A pilot study. In 2014 3rd International Conference on User Science and Engineering (i-USEr), pp. 216-220.

Dillon, G., \& Underwood, J. (2012). Computer mediated imaginative storytelling in children with autism. International Journal of Human-Computer Studies, 70, 169-178.

Dunn, R., Elgart, J., Lokshina, L., Faisman, A., Waslick, M., Gankin, Y., et al. (2017). Tablet-based cognitive exercises as an early parent-administered intervention tool for toddlers with autism-Evidence from a field study. Clinical Psychiatry, 3, 1-8. 
Higbee, T. S., Aporta, A. P., Resende, A., Nogueira, M., Goyos, C., \& Pollard, J. S. (2016). Interactive computer training to teach discrete-trial instruction to undergraduates and special educators in brazil: A replication and extension. Journal of Applied Behavior Analysis, 49(4), 780-793.

Lerman, D. V., Valentino, A. L., \& LeBlanc, L. A. (2016). Discrete trial training (pp. 47-83). Cham: Springer International Publishing.

Lovaas, O. I. (1987). Behavioral treatment and normal educational and intellectual functioning in young autistic children. Journal of Consulting and Clinical Psychology, 55(1), 3-9.

Maenner, M. J., Shaw, K. A., Baio, J., Washington, A., Patrick, M., DiRienzo, M., et al. (2020). Prevalence of autism spectrum disorder among children aged 8 years-Autism and developmental disabilities monitoring network, 11 sites, united states, 2016. MMWR. Surveillance Summaries, 69(4), $1-12$.

Manske, S., Werneburg, S., \& Hoppe, H. (2019). Learner modeling and learning analytics in computational thinking games for education, p. 187-212.

Medavarapu, S., Marella, L. L., Sangem, A., \& Kairam, R. (2019). Where is the evidence? a narrative literature review of the treatment modalities for autism spectrum disorders. Cureus.

Pimentel, E., Baldani, D., Piccolo, G., \& Hubner, M. (2009). An environment for the teaching of reading based on research in equivalence and control by minimum units. (Um Ambiente para o Ensino de Leitura baseado na Pesquisa em Equivalência e Controle por Unidades Mínimas). In XX Simpósio Brasileiro de Informática na Educação, Florianópolis-SC, Brasil. Original document in Portuguese.

Robacker, C., Rivera, C., \& Warren, S. (2016). A token economy made easy through classdojo. intervention in school and clinic. Intervention in School and Clinic, 52.

Sarokoff, R. A., \& Sturmey, P. (2004). The effects of behavioral skills training on staff implementation of discrete-trial teaching. Journal of Applied Behavior Analysis, 37(4), 535-538.

Siemens, G. \& Baker, R. S. J. d. (2012). Learning analytics and educational data mining: Towards communication and collaboration. In Proceedings of the 2nd International Conference on Learning Analytics and Knowledge, LAK'12, pp. 252-254, New York, NY, USA. Association for Computing Machinery.

Stenhoff, D. M., Pennington, R. C., \& Tapp, M. C. (2020). Distance education support for students with autism spectrum disorder and complex needs during covid-19 and school closures. Rural Special Education Quarterly, 39(4), 211-219.

Trevisan, D. F., Becerra, L., Highbee, T. S., Benitez, P., \& Gois, J. P. (2019). A review of the use of computational technology in applied behavior analysis. Adaptive Behavior.

Trevisan, D. F., Benitez, P., \& Gois, G. P. (2020). A web framework for configurable games with application to autistic children (pp. 520-527). Recife - PE - Brazil.: In Proceedings of SBGames 2020.

Trevisan, D. F., Benitez, P., Sandoval, P., \& Gois, J. P. (2018). Using the coji robot to teach pre-verbal abilities for an autistic student. ("Uso do Robô Coji Para Ensino de Habilidades Pré-verbais Para Estudante com Autismo). In Anais do 8 Congresso Brasileiro de Educação Especial. Original document in Portuguese.

Vivanti, G., \& Pellecchia, M. (2020). Applied behavior analytic approaches to early intervention for children with autism (pp. 79-91). Cham: Springer International Publishing.

World Health Organization (2020). International statistical classification of diseases and related health problems. 11th edition. Retrieved from https://icd.who.int/.

Yuan, J., Chen, C., Yang, W., Liu, M., Xia, J., \& Liu, S. (2020). A survey of visual analytics techniques for machine learning. Computational Visual Media.

Publisher's Note Springer Nature remains neutral with regard to jurisdictional claims in published maps and institutional affiliations. 

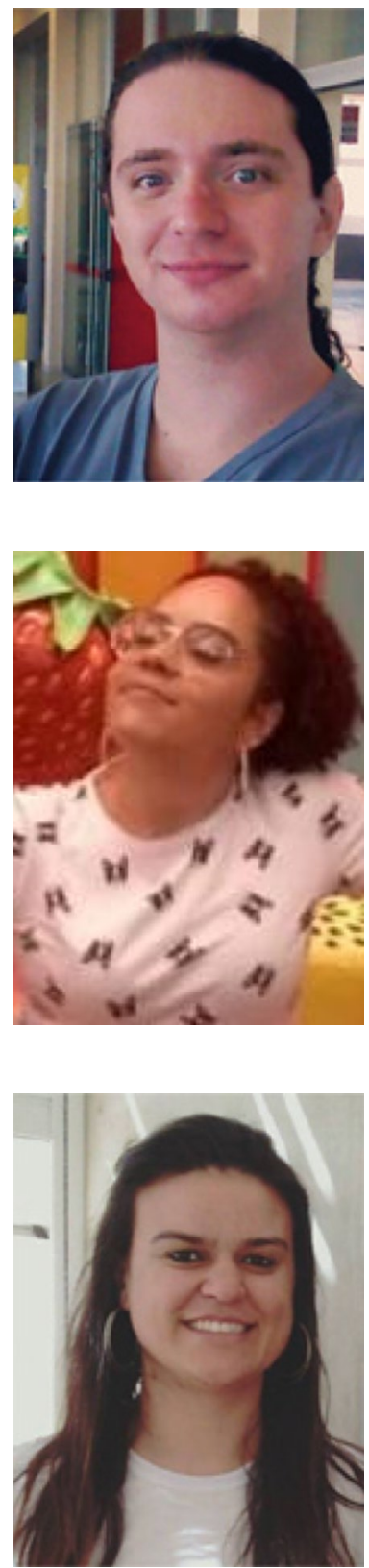

Diogo Fernando Trevisan is currently professor at State University of Mato Grosso do Sul and Ph.D. candidate at Federal University of $\mathrm{ABC}$ (UFABC). His main interests are interactive computer graphics, games and Human-Computer Interaction.

Fernanda A. B. de Araujo is an undergraduate student at Federal University of ABC (UFABC). Her interests are applied behavior analysis, autism,intellectual deficiency, and inclusive education.

Priscila Benitez is a professor at the Center of Mathematics, Computing, and Cognition of the Federal University of ABC (UFABC), Brazil. Her interests are applied behavior analysis, autism, intellectual deficiency, inclusive education, and family. 


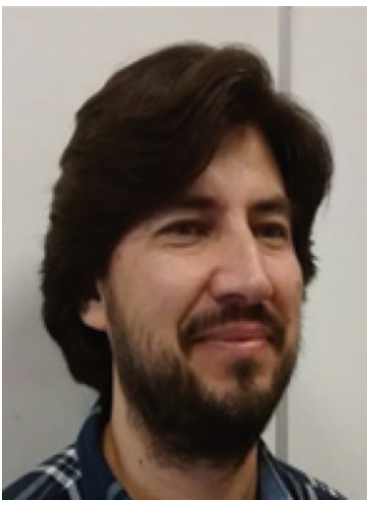

João Paulo Gois is an associate professor at the Center of Mathematics, Computing, and Cognition of the Federal University of ABC (UFABC), Brazil. His main interests are interactive computer graphics, geometric modeling, computer animation, and human-computer interaction. 